\title{
IAMJ
}

INTERNATIONAL

AYURVEDIC

MEDICAL JOURNAL

Review Article

ISSN: 2320-5091

Impact Factor: 6.719

\section{REVIEW OF LITERATURE OF RAMBAN RASA A HERBO MINERAL FORMULATION}

\author{
Sachin S Sheth ${ }^{1}$, Shardul P Chavan ${ }^{2}$, Kiran Sudhakar Darade ${ }^{3}$ \\ ${ }^{1}$ Assistant Professor, Dept of Rasashastra and Bhaishajya Kalpana; \\ ${ }^{2}$ Assistant Professor, Dept of Rasashastra and Bhaishajya Kalpana; \\ ${ }^{3}$ P. G. Scholar, Dept of Rasashastra and Bhaishajya Kalpana; \\ APM's Ayurved Mahavidyalaya, Sion, Mumbai-22, Maharashtra, India
}

Corresponding Author: daradeskiran15@gmail.com

\section{https://doi.org/10.46607/iamj2608122020}

(Published online: December 2020)

Open Access

(C) International Ayurvedic Medical Journal, India 2020

Article Received: 24/11/2020 - Peer Reviewed: 27/11/2020 - Accepted for Publication: 30/11/2020

\section{Check for updates}

\begin{abstract}
Rasashastra and Bhaishajya Kalpana is a branch of Ayurveda that deals with the pharmaceutical processing, preparation of compound medicines and therapeutic utility of herbal drugs, metals and minerals. Ramban Rasa is one such Kharaliya herbo-mineral formulation mentioned in various Ayurvedic texts. One formulation prepared by Sidhant of Pachan. Ramban Rasa is commonly indicated in Jwara Chikitsa (Fever). Even there are references to be used in disease such as Prameha (Diabetes mellitus), Agnimandya (Indigestion), Aamvata (Rhumatism), Ajirna (Indigestion), Grahani (Irritability bowel syndrome), Rasayana (Immunity booster), Vatvyadhi (Diseases of Vata). Variation found in Dravya, Bhavana dravya, size of formulation, Dose, Anupana, and Rogadhikar of Ramban Rasa among all classics. Compilation of these will help to study in comparative manner.
\end{abstract}

Keywords: Ramban Rasa, Kharaliya Rasayana, Jwara.

\section{INTRODUCTION}

Herbo-mineral compounds occupy significant seat in Ayurvedic pharmaceuticals. Rasaushadhis are the medicines of metallic and mineral origin.
"Alpamaatropayogitwaat Arucera Aprasangataha. Kshipram Aarogyadaayitwat Aushadhyebhyo Adhiko Rasahal" In current kinetic era, Rasaushadhies have 
given Ayurveda a complete novel health care approach. The innate qualities like quick action, lesser dose, tastelessness, prolonged shelf life, better palatability of Rasaushadhies have helped them to conquer the demand of patients as well as pharmaceutical proprietors ${ }^{1}$. Thus, the above all characteristics are seen in kharaliya rasayana which is combination of herbal drugs and metals- minerals triturated together along with liquid Dravyas. Ramban Rasa is one such Kharaliya rasayana which is indicated in Jwara. Jwara has been said to be the chief among the diseases (Rogānāma Pradhānah) ${ }^{2}$. Its importance has been established by the statement that it attacks the living body at its both the times the birth and the death. It is also said that there is no living body which is not attacked by it. The term Jwara is generally translated as fever, though it does not convey all the senses of the term. The term Jwara means that condition in which mind, sense organs and the body all are troubled (Dehendriya Mana Santaap) ${ }^{3}$. Jwara (Fever) is characterized by elevation of body temperature, loss of perspiration and varying degree of pain in the body. The Jwara as a disease has been clinically divided and subdivided under many groups. Thus, for management of this disease. Ramban Rasa has been indicated for various types of these diseases. There are various references available having name as Ramban Rasa in various Rasa Aushadhi text. Though variation in ingredients is observed among these, there is resemblance in almost all formulations in perspective of levigation. Thus, present study is an attempt to go through references of Ramban Rasa formulations in literature in order to have comparative better understanding of these formulations in terms of their composition, drugs specified for levigation, indications and contribution of various texts regarding this particular drug.

Aim

1. Detail literary study of Ramban rasa.

2. Detail literary study of contains and pharmaceutical procedure of Ramban rasa Chaturth.

Thus, the present study is an attempt to compile all available references of Ramban Rasa available in text and to study them in comparative manner in terms of their composition, method of preparation, dose, indications and contribution of various texts regarding this particular formulation.

\section{Materials and Methods}

References of Ramban Rasa will be compiled from various Rasa text and compendium and particular contribution of each text will be noted. Research works conducted at various institutes were not available hence published data was compiled to interpret the classical information.

\section{Literary Review}

Ramban Rasa is not mere a single formulation but a variety of formulations across different text have been quoted as Ramban Rasa. Name of drug itself throws light on the action of the compound i.e. it is a formulation which something that will cure all diseases (a universal remedy). Following citations have been found in text such as Bhaishajya Ratnavli and Rasaratna Samucchay. Other references have been collected from compendium Rasa Yog Sagar and Bharat bhaishajya ratnakar are listed below:

Table 1: Showing different ingredients of Ramban Rasa and various drugs used for trituration with Rogadhikar according to different Acharyas.

\begin{tabular}{|c|c|c|c|c|}
\hline Sr. No & Reference & Ingredients & Bhavana Dravya & Rogadhikar \\
\hline 1 & $\begin{array}{l}\text { R.R. } S^{4} \\
\text { R.Y.S }{ }^{5}-1 \\
\text { B.B.R }{ }^{6}-2\end{array}$ & $\begin{array}{l}\text { Raupya (vangmarit), Suvarna (Nag marit), Par- } \\
\text { ad bhasma, Gandhak, Tapya Bhasma, Vaikrant } \\
\text { bhasma, Rajavarta Bhasma, }\end{array}$ & $\begin{array}{l}\text { 1.Ankolmul kwath } \\
\text { 2. Ankol Beej } \\
\text { 3. Barbur Kwath }\end{array}$ & Prameha \\
\hline 2 & $\begin{array}{l}\text { R.Y. } S^{5}-2 \\
\text { B. } \mathrm{R}^{7} \\
\text { A.S. } \mathrm{S}^{8} \\
\text { R.P.Su }\end{array}$ & $\begin{array}{l}\text { Parad, Gandhak, Lavang, Vatsanabh, Marich, } \\
\text { Jatiphal }\end{array}$ & $\begin{array}{l}\text { Tintidik Phal } \\
\text { Rasa, Bijapura, } \quad \mathrm{Na}- \\
\text { rang, Dadim, } \quad \mathrm{Sa}- \\
\text { dakusum, Shrungber }\end{array}$ & Agnimandya \\
\hline 3 & R.Y. $S^{5}-3$ & Parad, Gandhak, Vatsanabh, Marich, Jaipala, & Tintidik Rasa & Jwar \\
\hline
\end{tabular}




\begin{tabular}{|c|c|c|c|c|}
\hline & B.B.R -1 & Hingul, Tankan & & \\
\hline 4 & R.Y.S $5^{5}-4$ & Tuttha, Gandhak, Hartal & Kanyadrava & Jwar \\
\hline 5 & R.Y.S $S^{5}-5$ & Tuttha, Rasak, Somal & Karvellak Rasa & Jwar \\
\hline 6 & R.Y.S $S^{5}-6$ & $\begin{array}{l}\text { Shwet kshar, Pit Kshar, Parad, Vanga bhasma, } \\
\text { Manashila, Gandhak, Shwet Khadir }\end{array}$ & Nagvalli Dal Rasa & Vatvyadhi \\
\hline 7 & R.Y.S $S^{5}-7$ & $\begin{array}{l}\text { Parad, Gandhak, Rasakarpur, Jatiphal, Trikatu, } \\
\text { Yavakshar, Vatsanabh }\end{array}$ & Nagvalli Dal & $\begin{array}{l}\text { Darun } \\
\text { Sannipat } \\
\text { Vishamjwar }\end{array}$ \\
\hline 8 & R.Y.S $S^{5}-8$ & $\begin{array}{l}\text { Parad, Tankan, Abhrak, Darad, Loha (Kant, } \\
\text { Tikshna), Tamra, Suvarna mauktik, Vidrum, } \\
\text { Gandhak, Raupya, Kasturi, Karpur, Jatiphal, } \\
\text { Lavang }\end{array}$ & $\begin{array}{l}\text { Shrikhand (S.chandan), } \\
\text { Triphala, Erandmul, } \\
\text { Kamal, Punnag, } \\
\text { Jambir, Hriber, Utpal, } \\
\text { Mallika, Kumud phal }\end{array}$ & $\begin{array}{l}\text { Rasayana, } \\
\text { Gulma, Pliha, } \\
\text { Bhagandar, } \\
\text { Jwar, Prameha, } \\
\text { Mutraroga, } \\
\text { Mutrakruccha }\end{array}$ \\
\hline 9 & R.Y. $S^{5}-9$ & $\begin{array}{l}\text { Jati, Lavang, Rasamanikya, Mushikapashan } \\
\text { (Somal) }\end{array}$ & Dhattur Rasa & Jwar \\
\hline 10 & R.Y. $S^{5}-10$ & Nilanjan, Tuttha, Gauripashan & $\begin{array}{l}\text { Dhatturpatra } \\
\text { Swaras }\end{array}$ & Jwar \\
\hline 11 & R.Y.S -11 & $\begin{array}{l}\text { Parad, Tuttha, Manashila, Hartal, Kharpar, } \\
\text { Marich }\end{array}$ & Jambir Ras & Jwar \\
\hline 12 & R.Y.S $S^{5}-12$ & $\begin{array}{l}\text { Nillanjan, Tuttha, Gauripashan, Kharpar, } \\
\text { ShwetPashan, Manashila, Hartal }\end{array}$ & Jambir Rasa & Jwar \\
\hline 13 & R. Y.S $S^{5}-13$ & Gauripashan, Tuttha, Sudha & Jambir Rasa & Jwar \\
\hline 14 & R.Y.S. ${ }^{5}-14$ & $\begin{array}{l}\text { Tankan, Hartal, Nilanjan, Parad, Vatsanabh, } \\
\text { Gandhak, Somal (Pit, Shwet), Rasanjan, Mana- } \\
\text { shila }\end{array}$ & $\begin{array}{l}\text { Jambir Rasa, Nirgundi } \\
\text { Swaras }\end{array}$ & Jwar \\
\hline 15 & R.Y.S. ${ }^{5}-15$ & $\begin{array}{l}\text { Parad, Gandhak, Tamra, Tankan, Tiphala, } \\
\text { Vatsanabh, }\end{array}$ & Karvella Rasa & Jwar \\
\hline 16 & R.Y.S. ${ }^{5}-16$ & $\begin{array}{l}\text { Hingul, Rasak, Parad, Tuttha, Hartal, Mana- } \\
\text { shila, Gandhak, Tapya, Somal }\end{array}$ & $\begin{array}{l}\text { Krushna } \\
\text { Dhattur }\end{array}$ & Sheet Jwar \\
\hline 17 & B.B.R ${ }^{6}-4$ & $\begin{array}{l}\text { Parad, Gandhak, Trikatu, Vatsanabha, Jaipala, } \\
\text { Tankan, Hingul }\end{array}$ & - & Jwar \\
\hline
\end{tabular}

(R.R. S-Rasa Ratna Samucchay, R.Y. S- Rasa Yog Sagar, B.B. R- Bharat Bhaishyya Ratnakar, B. RBhaishajya Ratnavali, A.S. S-Ayurved Sarsangraha, R.P.Su- Rasaprakash Sudhakar)

Indication of Ramban Rasa in various diseases - Various diseases related with the Rasavaha strotas, Mutravaha strotas, Swedovaha strotas, Annavaha strotas etc. are included in the indications. Text like Rasa Yog Sagar, Bharat Bhaishjya Ratnakar, Rasamanjiri, Rasayan Sangraha, Vaidya vilas, Rasakam dhenu, Rasa prakash, Vaidya chintamani, Ratnakaraushadhyog etc. have indicated various formulations to be indicated for Jwara in the name of Ramban Rasa. Rasaratna sammucchay mentions the drug under the treatment of Prameha (Diabetes mellitus), while Bhaishajya ratnavali, Ayurved sarasangraha and Rasaprakash sudhakar texts indicates the drug with appropriate Anupana in various diseases. 
Table 2: List of Matra and various Anupana with Pathya of Ramban Rasa according to different Acharyas:

\begin{tabular}{|c|c|c|c|c|}
\hline Sr. No & Reference & Matra & Anupana & Pathya \\
\hline 1 & $\begin{array}{l}\text { R.R. } S^{4} \\
\text { R.Y.S }{ }^{5}-1 \\
\text { B.B.R }{ }^{6}-2\end{array}$ & 3 Ratti & Giloy satva & - \\
\hline 2 & $\begin{array}{l}\text { R.Y. } S^{5}-2 \\
\text { B. } \mathrm{R}^{7} \\
\text { A.S. } \mathrm{S}^{8} \\
\text { R.P.Su }\end{array}$ & 1 Mash & $\begin{array}{l}\text { 1.Ardrak rasa- Kapha } \\
\text { 2.Nirgundi rasa- vata } \\
\text { 3.Dhanya Jala-Pitta } \\
\text { 4.Trikatu, Vasarasa-Shwas } \\
\text { 5.Shunthi, Saindhav, Haritaki-Udar } \\
\text { 6.Punarnava Kwath-Shoth } \\
\text { 7.Gomutra / Trikatu, Triphala kwath - Pandu } \\
\text { 8.Madhu- Kshaya } \\
\text { 9.Erand taila kwath-Visham/vatvedana. }\end{array}$ & - \\
\hline 3 & $\begin{array}{l}\text { R.Y. } S^{5}-3 \\
\text { B.B.R }{ }^{6}-1\end{array}$ & 1 Ratti & Tulsi Patra swaras- Sarva Vishamjwara & Dugdha, Odan, Dadhi \\
\hline 4 & R.Y.S $5^{5}-4$ & 3 Ratti (1 valla) & Sharkara- Jwar & Dugdha, Odan \\
\hline 5 & R.Y.S $5^{5} 5$ & Mudag matra & Gud-Chaturjatk Jwar & Dugdha, Odan \\
\hline 6 & R.Y.S 5 & Mudga mana & - & Mudga, Adhaki churna \\
\hline 7 & R.Y.S -7 & 1 Ratti & - & - \\
\hline 8 & R.Y.S $S^{5}-8$ & 4 Ratti & Mdhu, Sharkara, Dugdha & - \\
\hline 9 & R.Y. $S^{5}-9$ & 1 Gunja & Sharkara, Marich/Mudgsara(yush) & \\
\hline 10 & R.Y. $S^{5}-10$ & Marich & Sharkara, Dugdha & - \\
\hline 11 & R.Y.S $S^{5}-11$ & 1 Ratti & Jambir Rasa & \\
\hline 12 & R.Y.S $S^{5}-12$ & Mash & Sharkara, Jirak & \\
\hline 13 & R.Y.S ${ }^{5}-13$ & Chan & - & \\
\hline 14 & R.Y.S. ${ }^{5}-14$ & 6 Ratti & Sharkara, Madhu & Shali, \\
\hline 15 & R.Y.S. ${ }^{5}-15$ & 1 Gunjja & Aardak Ambu & \\
\hline 16 & R.Y.S. ${ }^{5}-16$ & 1Mash & - & - \\
\hline 17 & B.B. $R^{6}-4$ & - & - & - \\
\hline
\end{tabular}

Anupana for Ramban Rasa $14^{\text {th }}$ number formulation is Shali Odan, Mudgayush, Dadhi, Chach, Jangal mas, Ekshurasa, Kadali, Kharjur, Dadim, Kaittha. Ramban rasa chaturth is a unique preparation among all 16 references of Ramban rasa mentioned in Rasa Yog Sagar. It is the one of the formulations prepared ac- cording Siddhanta of Pachan which is indicated for Jwara vyadhi chikitsa. The ingredients are Shuddha Hartal, Shuddha Gandhak, Suddha Tuttha and Bhavna of Kumari swaras. Ramban rasa chaturth is separately compiled from Rasa Yog Sagar which is enlisted in Table

Table 3: Showing list of ingredients and their Quantity required of Ramban rasa chaturth ${ }^{10}$ mentioned in Rasa Yog Sagar.

\begin{tabular}{|l|l|l|l|}
\hline Sr. No & Ingredients & Name & Quantity \\
\hline 1 & Shuddha Tuttha & Copper sulphate $\left(\mathrm{CuSo}_{4} \mathrm{H}_{2} \mathrm{O}\right)$ & 3 parts \\
\hline 2 & Shuddha Gandhak & Sulphur $(\mathrm{S})$ & 5 parts \\
\hline 3 & Shuddha Hartal & Orpiment $\left(\mathrm{As}_{2} \mathrm{~S}_{3}\right)$ & 6 parts \\
\hline 4 & Ghritakumari Swaras & Aloe vera & As required \\
\hline
\end{tabular}

\section{Research Work ${ }^{11}$}

A research work naming Pharmaceutico Analytical Study of Shri Rambana Rasa published in Interna- tional Ayurvedic Medical Journal showed specific characteristics which are in accordance with the properties of Ramban Rasa. 


\section{DISCUSSION}

Total 17 references Ramban Rasa have been mentioned in Rasa Aushadhi compendium and text. Total 16 references from Rasa yoga sagar and 4 from Bharat Bhaishajya Ratnakar. As Acharya stated that "Roga sarvepi mandagnao". It means all Roga (Diseases) are caused due Agni Mandya (Poor Digestive fire). Agni mandya is also main root cause for Jwara vyadhi. Observing closely to each reference suggest that each Kalpa contains Dipan, Pachan and Agnivardhan dravyas. All the references given in the text shows that they mainly act on Annavaha strotasa. Rasadravyas can be used safely only after pharmaceutical processing. Pharmaceutical process includes Pachana as an important part. Pachana can be done by two types, Agni and Anagni Pachan. Pachan with Agni includes Kupipakva vidhi, Puta paka, Damru yantra, Musha etc. whereas Anagni pachan includes Kuttanam, Dalanam, Mardanam, Bhanjanam, Vilekhanam $^{12}$ etc. Thus, Agni plays a major role in formulation of every medicine. If we consider Kupipakva vidhi, almost all the Kupipakva rasayana kalpas mainly contain Parad as its main ingredient but Ramban Rasa Chaturth mentioned in Rasa Yog Sagar has exception.

\section{CONCLUSION}

It is clear from literature that Ramban Rasa is an emergency drug that is to be used in the systemic disorders such as different type of fever conditions, Diabetes mellitus even as rejuvenator. It also has special action in different diseases with specific Anupana. Thus, it has broad spectrum activity for the management of Kapha Vaataj disorders. As above mentioned, all ingredients mainly act as Agni Deepana, Pachana, Yakrututtejak, Rasayana, Krumighna. So, Ramban Rasa is effective in Jwara Vyadhi as a virtue of properties of all the ingredients.

The above formulation $4^{\text {th }}$ reference can be compared pharmaceutically by preparing in conventional method and muffle furnace.

\section{REFERENCES}

1. Narendranatha, Rasendra Sara Sangraha, $1 / 4-5, \mathrm{Pg}$ no. 2

2. Agniveshkruta, Charak Samhita, Acharya Vidhyadhar Shukla, $13^{\text {th }}$ edition, Chaoukhamba Publishing House, New Delhi, 2013.

3. Agniveshkruta, Charak Samhita, Acharya Vidhyadhar Shukla, $13^{\text {th }}$ edition, Chaoukhamba Publishing House, New Delhi, 2013, pg no. 73.

4. Acharya Shree Vagbhat, Rasa Ratna Samuchchaya, Edited by Dr. Indradev Tripathi, Chaukhambha Sanskrita Bhavan Prakashan, Varanasi (India), $3^{\text {rd }}$ edition, 17/76-80, Page no. 205

5. Vaidya Pandita Haripannaji Sharma, Rasa yoga Sagar, Published by Krushnadas Akadami, Varanasi (India), Part 2, Kalpa 169 to 184, Shlok 813 to 859, page no. 271-275.

6. Shah NC. Bharat Bhaishajya Ratnakar, Published by Jain Publishers, New Delhi (India) 2005, Part 4, Rasaprakaran, Kalpa 6146 to 6149 , Page no. 448 to 450 .

7. Govindadas, Bhaishajya Ratnavali. Mishra S (editor), Choukhamba Surabharati Prakashan; Varanasi, Reprint 2007,10/90-92, Page no. 341.

8. Sangrah AS. Published by Shri Baidhyanath Ayurved Bhawan Pvt. Ltd., Nagpur. 2004, Page no. 384.

9. Aacharya Yashodhar, Rasa Prakasha Sudhakar, Siddhiprada Hindi commentary by Dr. Siddhinandan Mishra, $2^{\text {nd }}$ edition 1998, Choukhamba Orientalia, Varanasi, 8/127-128, Page no.170.

10. Vaidya Pandita Haripannaji Sharma, Rasa yoga Sagar, Published by Krushnadas Akadami, Varanasi (India), Part 2, Kalpa 172, Shlok 827-828, page no. 272.

11. Gopi Krishna Maddikera et al: Pharmaceutico Analytical Study of Shri Rambana Rasa. International Ayurvedic Medical Journal \{online\} 2018 \{cited January, 2019\} Available from: http://www.iamj.in/posts/images/upload/39_44.pdf

12. Vaidya. P. V. Dhamanakar, Vaidya Gangadhar Puranik, Ayurvediya Aushadhikaran, Part 1 and 2, Shree Dhootapapeshwar Publication Pvt. LTD., $2^{\text {nd }}$ edition page no. 76 .

\section{Source of Support: Nil Conflict of Interest: None Declared}

How to cite this URL: Kiran Sudhakar Darade et al: Review Of Literature Of Ramban Rasa A Herbo Mineral Formulation. International Ayurvedic Medical Journal \{online\} 2020 \{cited December, 2020\} Available from: http://www.iamj.in/posts/images/upload/5359 5363.pdf 